\title{
Accuracy of point-of-care multiorgan ultrasonography for the diagnosis of pulmonary embolism
}

\author{
Peiman Nazerian ${ }^{1 *}$, Simone Vanni ${ }^{1}$, Giovanni Volpicelli ${ }^{2}$, Chiara Gigli ${ }^{1}$, Maurizio Zanobetti ${ }^{1}$, Alessandro Lamorte ${ }^{2}$, \\ Andrea Fabbri ${ }^{3}$, Stefano Grifoni ${ }^{1}$
}

From 9th WINFOCUS World Congress on Ultrasound in Emergency and Critical Care Hong Kong. 6-9 November 2013

\section{Background}

Presenting signs and symptoms of pulmonary embolism $(\mathrm{PE})$ are non-specific, favoring a large use of second-line diagnostic tests such as multi-detector computed tomography pulmonary angiography (MCTPA), thus exposing patients to high-dose radiation and to potential serious complications.

\section{Objective}

We investigated the diagnostic performance of multiorgan ultrasonography (lung, heart and leg veins ultrasonography) and if multiorgan ultrasonography combined to Wells score and D-dimer could safely reduce MCTPA tests.

\section{Patients and methods}

Consecutive adult patients suspected of PE and with a Wells score $>4$ or a positive D-dimer were prospectively enrolled in three emergency departments. Final diagnosis was obtained with MCTPA. Multiorgan ultrasonography was performed before MCTPA and considered diagnostic for $\mathrm{PE}$ if one or more subpleural infarcts, right ventricular dilatation or deep vein thrombosis were detected. If multiorgan ultrasonography was negative for $\mathrm{PE}$, an alternative ultrasonography diagnosis was searched for. Accuracies of each single-organ and multiorgan ultrasonography were calculated.

\section{Results}

PE was diagnosed in 110 (30.8\%) out of 357 enrolled patients. Multiorgan ultrasonography yielded a sensitivity of $90 \%$ and a specificity of $86.2 \%$, lung ultrasonography of $60.9 \%$ and $95.9 \%$, heart ultrasonography of $32.7 \%$ and $90.9 \%$ and vein ultrasonography of $52.7 \%$ and $97.6 \%$ respectively. Among the 132 (37\%) patients with multiorgan ultrasonography negative for PE plus an alternative ultrasonographic diagnosis or plus a negative D-dimer, no patients had PE as final diagnosis.

\section{Conclusions}

Multiorgan ultrasonography is more sensitive than single-organ ultrasonography, increases the accuracy of clinical pre-test probability estimation in patients with suspected PE and may safely reduce the MCTPA burden.

\section{Authors' details}

'Department of Emergency Medicine, Careggi University Hospital, Firenze, Italy. ${ }^{2}$ Department of Emergency Medicine, San Luigi Gonzaga University Hospital, Torino, Italy. ${ }^{3}$ Department of Emergency Medicine, Pierantoni Morgagni Hospital, Forlì, Italy.

Published: 31 January 2014

\section{doi:10.1186/2036-7902-6-S1-A25}

Cite this article as: Nazerian et al:: Accuracy of point-of-care multiorgan ultrasonography for the diagnosis of pulmonary embolism. Critical Ultrasound Journal 2014 6(Suppl 1):A25. 